\title{
RESPONSE TO EDITORIALS Resting-state brain functional MRI to complete the puzzle
}

\author{
Silvio Sarubbo, MD, PhD, ${ }^{1}$ Domenico Zacà, $\mathrm{PhD},{ }^{2}$ Lisa Novello, MSc, ${ }^{2}$ \\ Luciano Annicchiarico, MD, ${ }^{1,3}$ Francesco Corsini, MD, ${ }^{1}$ Umberto Rozzanigo, MD, ${ }^{4}$ \\ Franco Chioffi, MD, ${ }^{1}$ and Jorge Jovicich, $\mathrm{PhD}^{2}$
}

'Division of Neurosurgery, Structural and Functional Connectivity Lab Project, and ${ }^{4}$ Department of Radiology, Neuroradiology
Unit, "S. Chiara" Hospital, Trento; ${ }^{2}$ Center for Mind/Brain Sciences, University of Trento; and ${ }^{3}$ Department of Neurosciences,
Biomedicine and Movement Sciences, Section of Neurosurgery, University of Verona, Italy

$\mathrm{W}$ E are grateful to the authors of the 2 editorials on clinical resting-state functional MRI (rs-fMRI) for their positive and constructive comments about the paper published by our group in this issue of the Journal of Neurosurgery. These editorials give us the chance to further highlight limitations and perspectives of the approach we proposed.

Resting-state fMRI is an established and consolidated technique for the exploration of neural networks at the whole-brain level in human neuroscientific research. ${ }^{11}$ As a consequence, many research groups have provided valuable contributions for the wide use of this method in clinical and surgical fields, as masterfully highlighted by Dr. Smyth and Dr. Roland in their editorial. In fact, rs-fMRI has potentially opened a new door in clinical neurosciences, providing a unique chance to observe large functional frameworks at the whole-brain level with clinically acceptable data acquisition times. In particular, since rs-fMRI does not require the performance of a specific cognitive task, the method can be easily implemented in hospital settings and used regardless of the cognitive performance status of patients or their ability to cooperate, making it possible to even study brain networks of patients under anesthesia.

However, as we discussed in the paper, we are aware that rs-fMRI maps require further future validations, especially with respect to different rs-fMRI data acquisition and processing techniques. To this aim, the rs-fMRI tool that we are making available is based on consolidated rsfMRI data analysis methods that, to the best of our knowledge, have been shown to provide robust results on image data sets acquired with multivendor systems. In fact, the rs-fMRI data preprocessing steps applied in ReStNeuMap mostly replicate the pipeline described by Minati et al. ${ }^{10}$ This pipeline was later used in an rs-fMRI multisite study (13 3-T MRI centers) and provided consistent results across sites using different MRI hardware from different manufacturers. ${ }^{9}$ Likewise, the automatic template- matching procedure to label the rs-fMRI networks uses the goodness-of-fit (GOF) metric proposed in Van Dijk et al. ${ }^{15}$ In that original study, the GOF metric was applied to rs-fMRI data acquired using MRI systems with different static magnetic field strengths $(1.5 \mathrm{~T}$ and $3 \mathrm{~T})$ and manufacturers (Siemens and General Electric) to select the best-fitting component to sensorimotor, language, and visual templates. The rs-fMRI data reported in our paper are limited to the use of a 1.5-T MRI GE scanner and to maps derived from a small group of patients with different lesions. We fully agree with the comments in the editorial; the accumulation of a greater number of patients and a wider variety of tumors, MRI systems, and MRI acquisition protocols will be crucial to expand the findings of our first work. We hope that the free distribution of our ReStNeuMap analysis tool will help toward this aim, which was beyond the scope of this first study.

Moreover, since the first draft of this paper, we were very cautious to propose rs-fMRI as a method for intraoperative guidance during resections of brain lesions in eloquent areas. Our main goal was to develop, validate, and make available an automatic, updated, and updatable rs-fMRI analysis tool. This tool is meant to provide neurosurgeons with information about the functional processing of their patients at the whole-brain level and noninvasively, in line with the technical advancements available from the neuroscientific world. Precisely in this light, as extensively discussed in the paper, we decided to compare the functional MRI data obtained with ReStNeuMap with that obtained from direct electrical stimulation (DES), which is considered the most reliable tool (in terms of specificity and sensitivity) for the exploration of human brain function in neurosurgical practice, and it is a mandatory technique for safe resection of lesions in eloquent areas. $2,6,7,13$ As we reported, we found good consistency between the functional mapping findings from rs-fMRI and DES, with probabilistic values in the same range as reported in previous and larger comparisons. ${ }^{3}$ Therefore, 
our results contribute to the body of work that supports the validity of the information provided by rs-fMRI data. However, the intrinsic limitations of rs-fMRI do not make it yet a suitable replacement of other methods of functional mapping in awake surgery, such as DES. ${ }^{12}$

Regarding the limitations of our work, we also appreciate a comment made by Dr. Orringer in his letter (i.e., the lack of comparison between resting-state and task-based fMRI). We acknowledged, in fact, this weakness in our paper. Nevertheless, multiple studies have demonstrated that the sensorimotor, visual, and language rs-fMRI networks have topographies that are similar to those corresponding task-based fMRI activation maps. ${ }^{1,14}$ In addition, the spatial correspondence of functional maps provided by the 2 techniques is never complete because they measure different features of brain function..$^{5,8}$ Task-based fMRI is related to the performance of a task or administration of a stimulus, and the resulting maps represent the brain areas that are directly or indirectly involved in the task. In contrast, rs-fMRI refers to the brain's intrinsic activity, the degree of functional correlation between cortical and subcortical activity, and the resulting maps represent networks of synchronous BOLD activity. ${ }^{4}$ However, it has been shown that the degree of correspondence between the localization of preoperative functional activations, of both rs-fMRI and task-based fMRI, with intraoperative recording of functional responses by DES did not show significant differences between these 2 neuroimaging techniques. ${ }^{12}$ Therefore, given the possibility of an easy and wider use of rs-fMRI in clinical settings, we are focusing on developing, validating, and distributing a robust rs-fMRI analysis tool.

Finally, we would like to add a few comments, hopefully opening some new perspectives. The neurosurgical community is now in the unique position to have once again in its hands the chance to test, adopt, and possibly improve one of the significant technical advancements in the last 20 years of neuroscientific research. From this perspective, our efforts become a must. The functional information of the brain derived by rs-fMRI has some unique values that could be useful in our departments. Resting-state fMRI data should be, from our perspective, complementary and not alternative to other and well-established tools already included in our practice. Furthermore, rs-fMRI data may be useful not exclusively to complement preoperative planning but also to assess the effects of our treatments, and even more to observe the functional reorganization over the follow-up, especially in some fields of our practice, such as neuro-oncology, vascular surgery and brain trauma, and others (e.g., neurology, intensive care, radiotherapy, oncology, etc.).

To conclude, our work had 2 main goals. The first was to provide a user-friendly, testable, and updatable rs-fMRI analysis tool that may help us to comprehend, at the individual level, the functional assessment of patients in treatment planning as well as in evaluating the effects of those and the posttreatment plasticity. Second, as interestingly highlighted by Roland and Smyth in their editorial, we are hopefully contributing to strengthen the bridge between the neurosurgery and neuroscience worlds, to mutually improve knowledge and experiences of these 2 inseparable fields. We hope that such multidisciplinary efforts will contribute to taking us beyond the challenges of treating and evaluating brain diseases.

\section{References}

1. Calhoun VD, Kiehl KA, Pearlson GD: Modulation of temporally coherent brain networks estimated using ICA at rest and during cognitive tasks. Hum Brain Mapp 29:828-838, 2008

2. Chang EF, Breshears JD, Raygor KP, Lau D, Molinaro AM, Berger MS: Stereotactic probability and variability of speech arrest and anomia sites during stimulation mapping of the language dominant hemisphere. J Neurosurg 126:114-121, 2017

3. Cochereau J, Deverdun J, Herbet G, Charroud C, Boyer A, Moritz-Gasser S, et al: Comparison between resting state fMRI networks and responsive cortical stimulations in glioma patients. Hum Brain Mapp 37:3721-3732, 2016

4. Deco G, Corbetta M: The dynamical balance of the brain at rest. Neuroscientist 17:107-123, 2011

5. Dierker D, Roland JL, Kamran M, Rutlin J, Hacker CD, Marcus DS, et al: Resting-state functional magnetic resonance imaging in presurgical functional mapping: sensorimotor localization. Neuroimaging Clin N Am 27:621-633, 2017

6. Duffau H: New concepts in surgery of WHO grade II gliomas: functional brain mapping, connectionism and plasticity-a review. J Neurooncol 79:77-115, 2006

7. Duffau H, Lopes M, Arthuis F, Bitar A, Sichez JP, Van Effenterre R, et al: Contribution of intraoperative electrical stimulations in surgery of low grade gliomas: a comparative study between two series without (1985-96) and with (1996-2003) functional mapping in the same institution. J Neurol Neurosurg Psychiatry 76:845-851, 2005

8. Hou BL, Bhatia S, Carpenter JS: Quantitative comparisons on hand motor functional areas determined by resting state and task BOLD fMRI and anatomical MRI for pre-surgical planning of patients with brain tumors. NeuroImage Clin 11:378-387, 2016

9. Jovicich J, Minati L, Marizzoni M, Marchitelli R, Sala-Llonch R, Bartrés-Faz D, et al: Longitudinal reproducibility of default-mode network connectivity in healthy elderly participants: a multicentric resting-state fMRI study. Neuroimage 124:442-454, 2016

10. Minati L, Chan D, Mastropasqua C, Serra L, Spano B, Marra $\mathrm{C}$, et al: Widespread alterations in functional brain network architecture in amnestic mild cognitive impairment. J Alzheimers Dis 40:213-220, 2014

11. Preti MG, Bolton TA, Van De Ville D: The dynamic functional connectome: state-of-the-art and perspectives. Neuroimage 160:41-54, 2017

12. Rosazza C, Aquino D, D'Incerti L, Cordella R, Andronache $\mathrm{A}$, Zacà D, et al: Preoperative mapping of the sensorimotor cortex: comparative assessment of task-based and restingstate fMRI. PLoS One 9:e98860, 2014

13. Sanai N, Berger MS: Recent surgical management of gliomas. Adv Exp Med Biol 746:12-25, 2012

14. Smith SM, Fox PT, Miller KL, Glahn DC, Fox PM, Mackay $\mathrm{CE}$, et al: Correspondence of the brain's functional architecture during activation and rest. Proc Natl Acad Sci U S A 106:13040-13045, 2009

15. Van Dijk KR, Hedden T, Venkataraman A, Evans KC, Lazar SW, Buckner RL: Intrinsic functional connectivity as a tool for human connectomics: theory, properties, and optimization. J Neurophysiol 103:297-321, 2010

\section{Correspondence}

Silvio Sarubbo: silviosarubbo@gmail.com.

\section{INCLUDE WHEN CITING}

Published online October 26, 2018; DOI: 10.3171/2018.6.JNS181568. 